\title{
Bronchial artery embolization. What further we can offer?
}

\author{
Mohit Tayal ${ }^{1}$, Udit Chauhann ${ }^{1}$, Pankaj Sharma ${ }^{1}$, Rahul Dev ${ }^{1}$, Ruchi Dua ${ }^{2}$, Subodh Kumar ${ }^{3}$ \\ ${ }^{1}$ Division of Interventional Radiology, Department of Radiology, All India Institute of Medical Sciences, Rishikesh, India \\ 2Department of Pulmonary Medicine, All India Institute of Medical Sciences, Rishikesh, India \\ ${ }^{3}$ Department of Emergency Medicine, All India Institute of Medical Sciences, Rishikesh, India
}

Videosurgery Miniinv 2020; 15 (3): 478-487

DOI: https://doi.org/10.5114/wiitm.2019.89832

\begin{abstract}
Introduction: Massive hemoptysis is the most feared of all respiratory emergencies, with many underlying causes. In $90 \%$ of cases, the source of hemoptysis is the bronchial circulation. Despite high recurrence rates, bronchial artery embolization (BAE) remains the first-line treatment in management of hemoptysis.

Aim: To establish pre-procedure and procedural protocols for $B A E$.

Material and methods: The study included a total of 50 patients referred to the Department of Radiology for complaints of hemoptysis. Pre-procedure computed tomography (CT) angiography for determination of responsible circulation was performed as a regular protocol except in cases presenting with life-threatening hemoptysis. Polyvinyl alcohol (PVA size, 300-500 $\mu \mathrm{m}$ and 500-700 $\mu \mathrm{m}$ ) particles combined with gel foam embolization was performed. Successful catheterization and embolization of the targeted vessel was considered technical success and the cessation of hemoptysis to minimal levels was labeled clinical success.

Results: Thirty-two (64\%) male and 18 (36\%) female subjects comprised the study group. Forty (80\%) patients had moderate to severe hemoptysis. Tuberculosis (80\%) was the most common etiology. Five patients had severe AV shunting and were embolized with decremental particle size (500-700 $\mu \mathrm{m}$ followed by 300-500 $\mu \mathrm{m}$ ) to occlude the bed and then embolize the artery. Technical success was achieved in all the patients, but clinical success was achieved in 40 (80\%) patients.

Conclusions: Bronchial artery embolization is a minimally invasive procedure recognized for primary management of hemoptysis. Preprocedure evaluation with CT angiography can add incremental value in management. Usage of decremental particle size is helpful to embolize large AV shunts.
\end{abstract}

Key words: embolization, hemoptysis, bronchial artery, polyvinyl alcohol particles.

\section{Introduction}

Bronchial artery embolization (BAE) has gradually evolved in terms of technique, advanced hardware and newer embolic materials ever since its first description in 1974 by Remy et al. [1]. The procedure has an unequivocal role in palliative care for management of hemoptysis in patients who are not candidates for definitive treatment such as surgery. It is frequently done as an emergency procedure and offered to patients presenting with moderate to massive hemoptysis. Few authors have described its efficacy in controlling mild hemoptysis as well. The mortality in such cases is ascribed to asphyxiation from the aspiration of blood causing airway obstruction [2-4]. The etiologies contributing to hemoptysis are varied and range widely in different parts of the world. In the Asian subcontinent common causes include active/reactivation tuberculosis, post-tubercular sequelae, bronchiectasis and aspergillosis; on the

\section{Address for correspondence}

Udit Chauhan MBBS, MD, DNB, PDCC, Division of Interventional Radiology, Department of Radiology, All India Institute of Medical

Sciences, Rishikesh, India, e-mail: dr.udit.chauhan@gmail.com 
other hand, in western countries sarcoidosis, vasculitis and cystic fibrosis are known to be common culprits. Lung malignancies form a minority subgroup among etiologies in either population. Severity of hemoptysis and rate and frequency of recurrence are factors that, although independent of the cause, are important considerations that determine the clinical outcome of transcatheter therapies. Other factors that determine the technical/clinical success include the extent of lung involvement, presence of non-bronchial systemic feeders, tortuous aberrant bronchial circulation and bronchopulmonary shunts. A meticulous preprocedure evaluation with computed tomography (CT) angiography(CTA)/ fiberoptic bronchoscopy (FOB) is thus of paramount importance, although the tools are yet to find a position in the established protocol. CT angiography is comparable to FOB in lateralizing the bleeding, but is better suited for detection of the cause and is able to lay down a vascular roadmap before endovascular interventions [2, 5].

The aim of the present study is to investigate whether inclusion of CT angiography in the regular protocol for evaluation prior to embolization benefits the technical/clinical outcome. We tried to compare the results of using polyvinyl alcohol (PVA) particles along with gel foam with that of authors who have preferred using glue for embolotherapy. In addition, we have also attempted to highlight techniques of arteriography and embolization of responsible bronchial and non-bronchial circulation as well as associated procedural complications. We also share our experience with usage of decremental particle sizes in treatment of large AV shunts (between bronchial and pulmonary circulations) with associated hemoptysis.

\section{Aim}

The aim of the study was to establish pre-procedure and procedural protocols for BAE.

\section{Material and methods}

The Institutional Research and Ethical Committee approved this retrospective study that included cases of BAE referred to the Department of Radiology between July 2017 and December 2018. Written informed consent prior to the procedure was obtained from all of the subjects. A total of 50 patients, in an age range of 19 to 62 years, compris- ing 32 males and 18 females, comprised the study group.

A pre-procedure evaluation comprising comprehensive evaluation of symptoms, cause, and extent of lung involvement was performed in all patients. Hemoptysis was graded into mild, moderate and significant. It was categorized as mild when hemoptysis was less than $100 \mathrm{ml} /$ day or less than $50 \mathrm{ml} /$ episode, moderate when there was a single event of 100-300 ml/day or $>3$ bouts of more than $100 \mathrm{ml} /$ day in 1 week. Significant grade was hemoptysis of more than $300 \mathrm{ml} /$ day or any amount leading to more than $1 \mathrm{~g} / \mathrm{dl}$ hemoglobin drop or more than a 5\% hematocrit drop. CTA before the procedure was performed in all the patients except two, who presented with life-threatening hemoptysis and were taken directly for embolization. Transfemoral was entry route in all patients.

A $5 \mathrm{Fr}$ angiographic sheath (Boston Scientific, Boston, MA, USA) was placed in the common femoral artery. Through the sheath, a 4/5-French Slip-cath Beacon tip Cobra catheter (Cook Medical, Bloomington, IN, USA) was used to catheterize the orthotopic bronchial arteries, which were cannulated around the region of the carina/left main bronchus in the anterior angulation. When cannulation with a Cobra catheter was not possible SIM-1 (Simmons) or Shepherd hook (Boston Scientific, Boston, MA, USA) catheters were used. After catheterization of the bronchial artery an angiogram (2 frames per second) was performed using iobitridol $(350 \mathrm{mg} / \mathrm{ml}$ Xenetix, Guerbet, France) to identify the abnormal (> $2 \mathrm{~mm}$, hypertrophied and tortuous course) bronchial arteries. Following the identification and affirmation of the abnormal bronchial artery it was superselectively cannulated with a 2.7 Fr microcatheter (Progreat Terumo, Somerset, NJ, USA) with the tip of catheter placed and secured distal to the ostia of intercostal/spinal arteries. Repeat angiogram was done from the microcatheter to confirm the position. A road map was used whenever needed while facing any difficulty in superselective cannulation. PVA particles (Cook Medical, Bloomington, IN, USA) of size 300-500 $\mu \mathrm{m}$ and 500-700 $\mu \mathrm{m}$ along with gel foam (Upjohn, Kalamazoo, MI, USA) were used to embolize the responsible circulation. The primary end point was reached when the column of contrast after embolization was seen to stay for at least five heart beats. Repeat contrast injection was made after a wait of 2-3 min. In patients with large AV 
shunts (between bronchial and pulmonary arteries) decremental size of PVA particles was used. Initial bed embolization was done with 500-700 $\mu \mathrm{m}$ particle size followed by embolization of the artery with 300-500 $\mu \mathrm{m}$ size particles.

The outcomes of the procedure were categorized into technical and clinical success. Successful catheterization and embolization of the targeted vessel was considered technical success and the stoppage or reduction of hemoptysis to minimal levels was labeled clinical success.
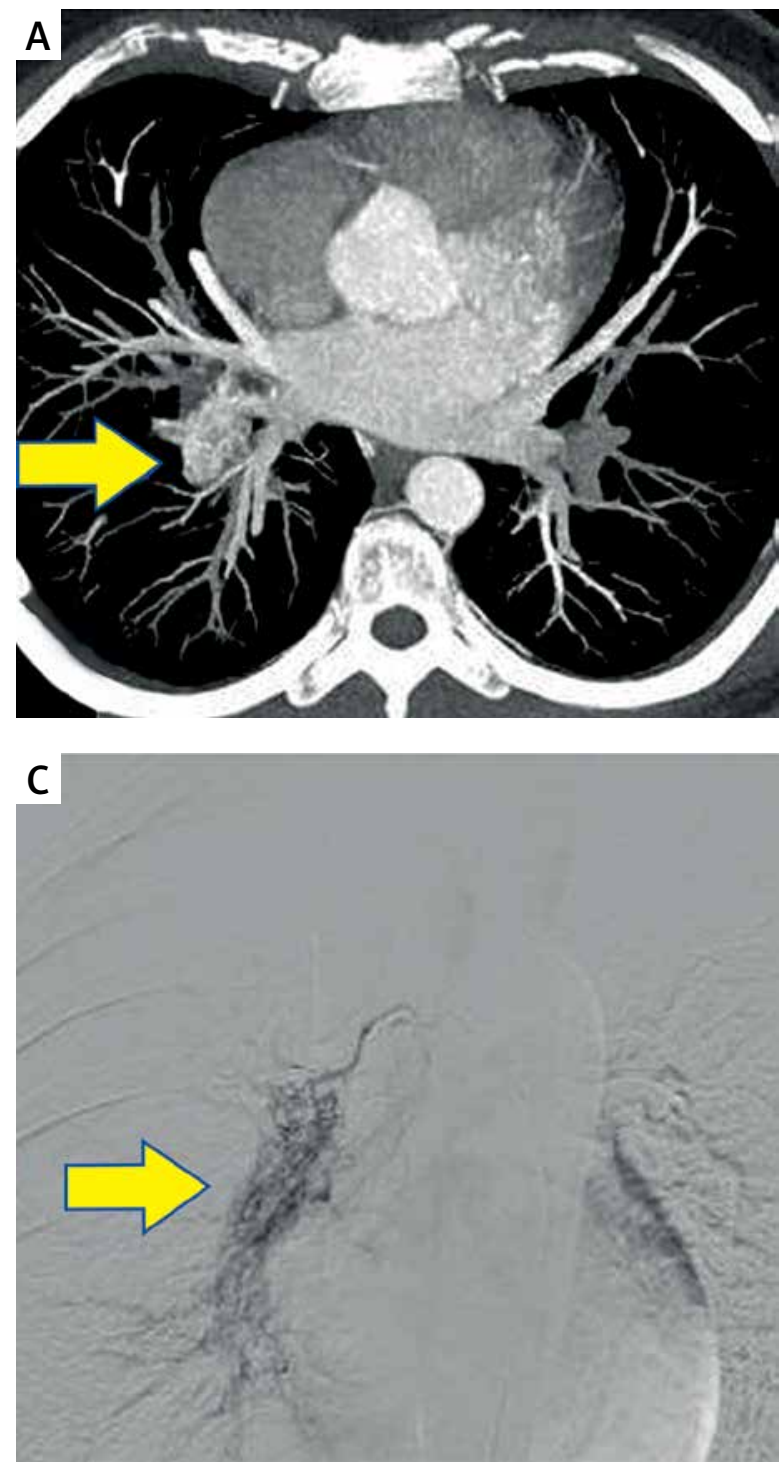

\section{Results}

A total of 50 patients, in an age range of 19 to 62 years, comprising 32 males and 18 females, comprised the study group. Of the total 50 patients, 40 patients had active/reactivation of tuberculosis. Four patients were detected to have aspergillosis, 1 had lung malignancy. There was 1 patient each with bronchial varices (Photo 1) and pulmonary hamartoma. In 3 patients, the etiology could not be ascertained. Eighty percent $(n=40)$ of patients presented with moderate to significant hemoptysis,
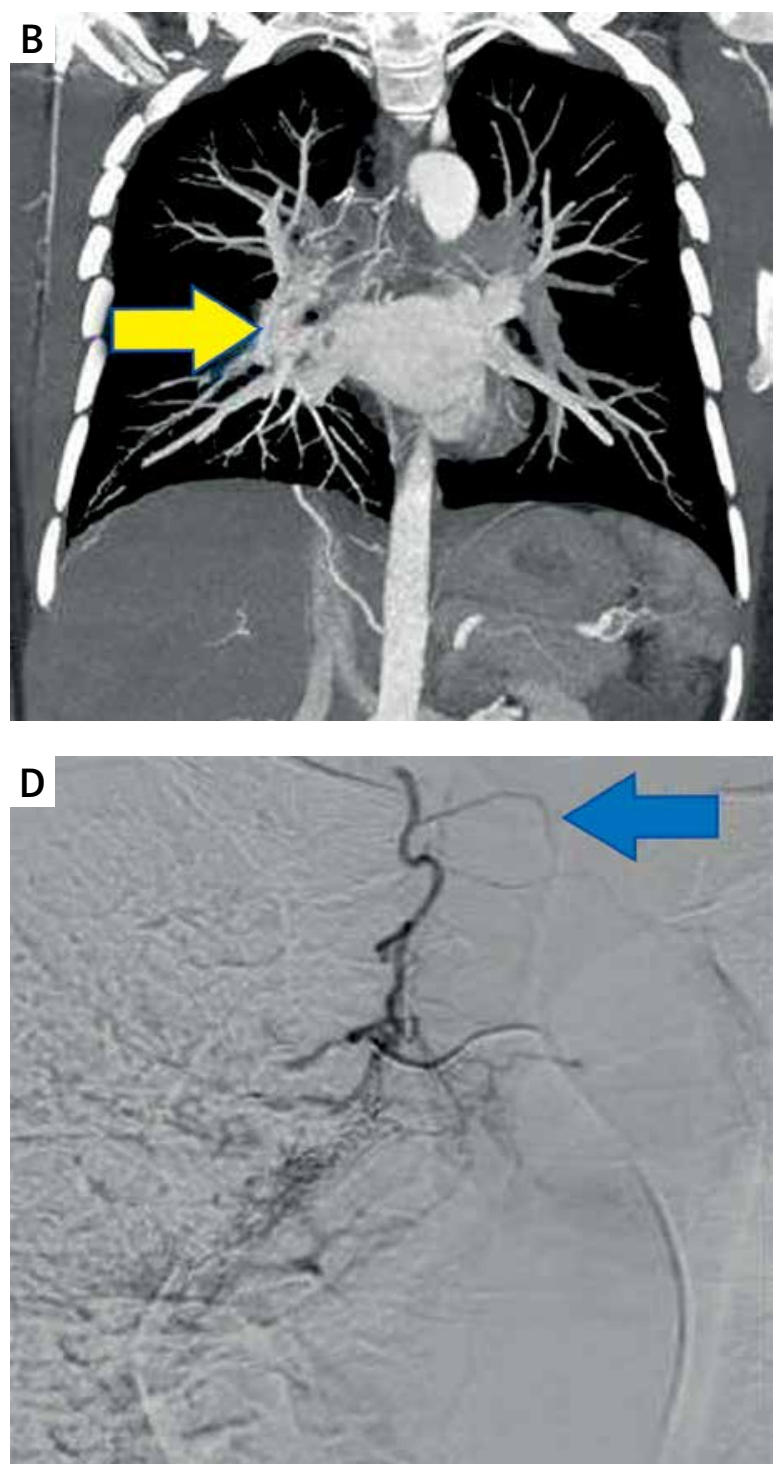

Photo 1. A, B - Multiplanar MIP reconstruction images showing tuft of tortuous dilated vessels (arrows) along the right lower lobe bronchus, confirmed to be bronchial varices on digital subtraction images (C). D - Another DSA image in the patient showing origin of anterior spinal artery with the classical hairpin configuration 
and $20 \%(n=10)$ of patients had mild hemoptysis at the time of presentation. Pre-procedure CTA was performed in all the patients, except two. Technical success was achieved in all the patients. Clinical success was attained in $90 \%(n=45)$ of patients. Ten $(20 \%)$ patients had recurrence, of which none had a bleeding episode within 15 days, 5 had episodes within 1 month and the other 5 had recurrence after 3 months. Among post-procedure complications, transient chest/back pain was noted in $28 \%(n=14)$ of patients. Dysphagia was reported by $1(2 \%)$ patient. Transverse myelitis was observed in $1(2 \%)$ patient.

\section{Discussion}

\section{Anatomical considerations}

The bronchial and pulmonary arteries in combination form the blood supply to the lungs, wherein the pulmonary artery is responsible for approximately $99 \%$ of perfusion. Bronchial arteries supply the bronchi and lung interstitium, and also contribute to supply of the posterior mediastinum, vagus nerve, trachea and esophagus. The elaborate knowledge of anatomy and variability is important to minimize the risk of otherwise inadvertent complications.

Bronchial arteries typically arise from descending thoracic aorta between T3-T8 vertebral bodies. The majority $(\sim 70 \%)$ of times they arise at the T5T6 vertebral level and the left main bronchus forms an important landmark on fluoroscopy. Various anatomic permutations have been described in the literature regarding the origin and branching pattern of bronchial arteries arising from the aorta, of which the classical four configurations were illustrated by Cauldwell et al. [6]. Type 1 and type 2 configurations consist of a solitary right bronchial artery arising from an intercostobronchial trunk in conjunction with two and single left bronchial arteries respectively. Type 3 configuration has two bronchial arteries on either side, one of the right bronchial artery arising in conjunction with an intercostobronchial trunk. In type 4, there are two bronchial arteries on the right side, of which one arises from an intercostobronchial trunk with a solitary left bronchial artery (Figure 1). Another less commonly reported configuration is the presence of a common trunk from which there arises one bronchial artery on either side $[2,7]$. In agreement with the available literature, our experience also suggests type 1 ( $n=23 ; 46 \%)$ as the most frequent observation, but it was followed closely by presence of a common arterial trunk ( $n=$ $19 ; 38 \%)$. Type $2(n=5 ; 10 \%)$ and type $3(n=3 ; 6 \%)$ permutations were very infrequently encountered. Non-bronchial systemic feeders and aberrant bronchial vessels are not infrequent findings in diseased lung, and when present, their embolization is necessary to achieve good clinical outcome. These have always been a concern to interventionists when doing a pre-procedural evaluation. They may arise from almost any thoracic and abdominal arteries, the most common being subclavian, internal mammary, thyrocervical and superior intercostals arteries. It is essential to differentiate bronchial from systemic collaterals through close inspection of the relationship of the vascular course with that of the bronchial tree. Both ectopic and orthotopic bronchial arteries follow the course of the associated bronchi, whereas systemic nonbronchial collateral arteries are never seen joining the bronchial tree. In the present study, we found ectopic bronchial arteries in $24 \%(n=12)$, non-bronchial systemic collaterals in 10\% $(n=5)$
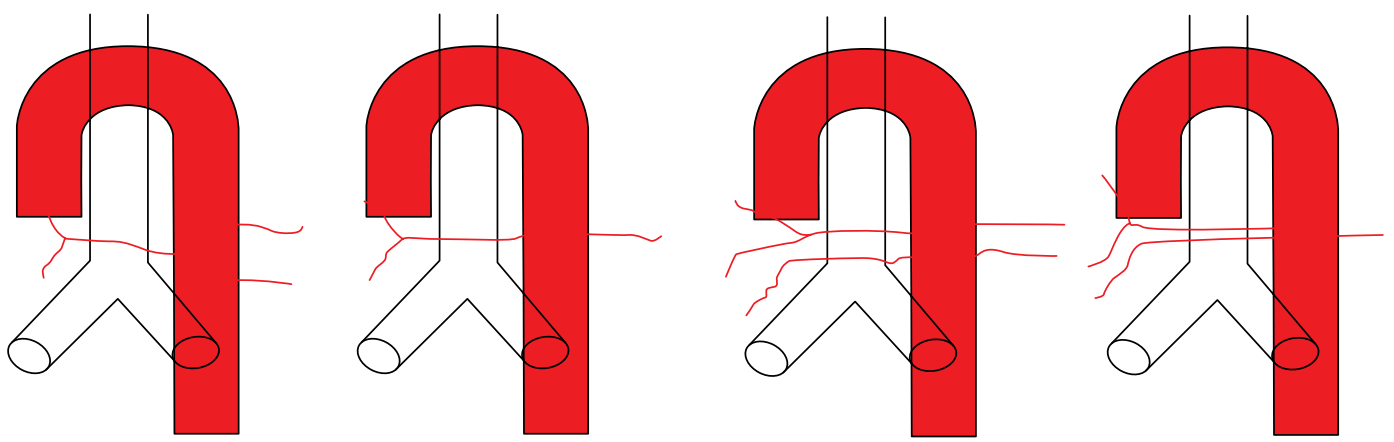

Figure 1. Line diagram depicting common types of bronchial artery configurations (type I to type IV; left to right) described by Cauldwell et al. [6] 

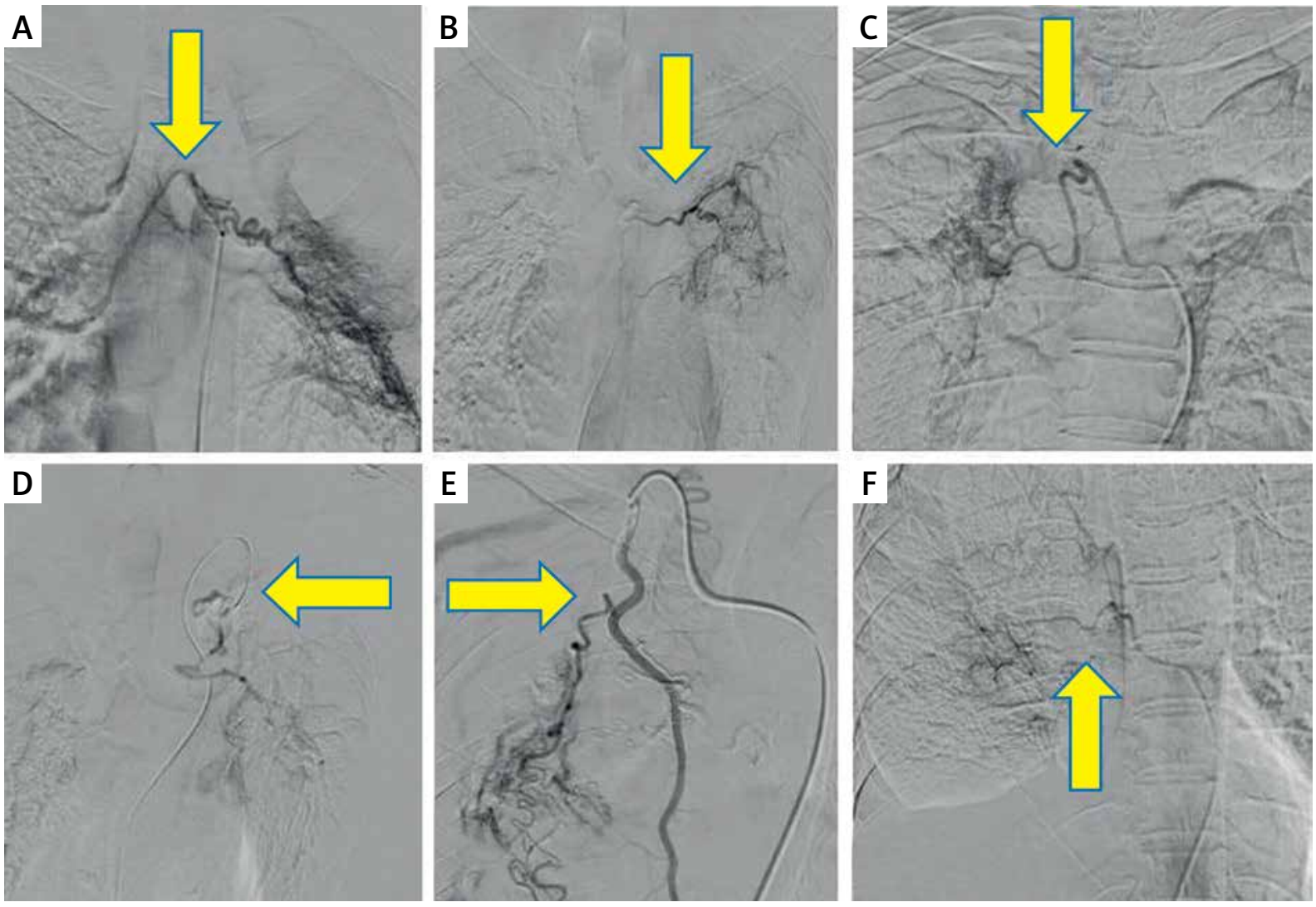

Photo 2. Various anatomical permutations of responsible circulation found in the present study. A, B - Depicting origin of right and left bronchial arteries from the descending aorta with an independent origin. C - Showing a right intercosto-bronchial trunk. D - Aberrant bronchial circulation arising from the left internal mammary artery. E - Non-bronchial systemic circulation arising from internal thoracic artery on right side. $\mathbf{F}$ - Showing parenchymal blush in right lung parenchyma due to extravasation from an intercostal artery

and presence of bronchopulmonary shunts in $14 \%$ $(n=7)$ of cases (Photos 2 and 3).

The anterior spinal artery courses along the ventral surface of the cord and receives supply from segmental medullary arteries. Classically, these are described to have "hairpin" configuration on angiography. The artery of Adamkiewicz, arising from an intercostal artery, is most commonly seen at the T8-L1 vertebral level [8]. It was encountered in 6\% $(n=3)$ of cases in the present study and non-target embolization was successfully avoided in two of the three cases.

\section{Pre-procedure evaluation}

Grading of hemoptysis into mild, moderate and severe has been differently described in the literature depending on the volume and duration of bleeding [9-11]. Being a tertiary care health center, we had a relatively skewed case distribution with more cases of moderate to massive hemoptysis ( $n=40 ; 80 \%$ ) undergoing BAE compared to only $20 \%$ $(n=10)$ of cases presenting with mild hemoptysis.

The utility of pre-procedure diagnostic work-up with CTA or FOB has been reiterated in the literature by various authors. Whereas results of both CTA and FOB are comparable in lateralizing of bleeding, CTA fares better in being more informative about the cause, extent of lung involvement and presence of aberrant bronchial and systemic non-bronchial circulation [2, 9, 12-14]. We were able to perform CTA in nearly all cases except two those presented with life-threatening hemoptysis. Good clinical outcomes observed in the present study can be attributed to CTA done prior to the procedure. In $26 \%(n=13)$ of cases, responsible circulation could not be detect- 

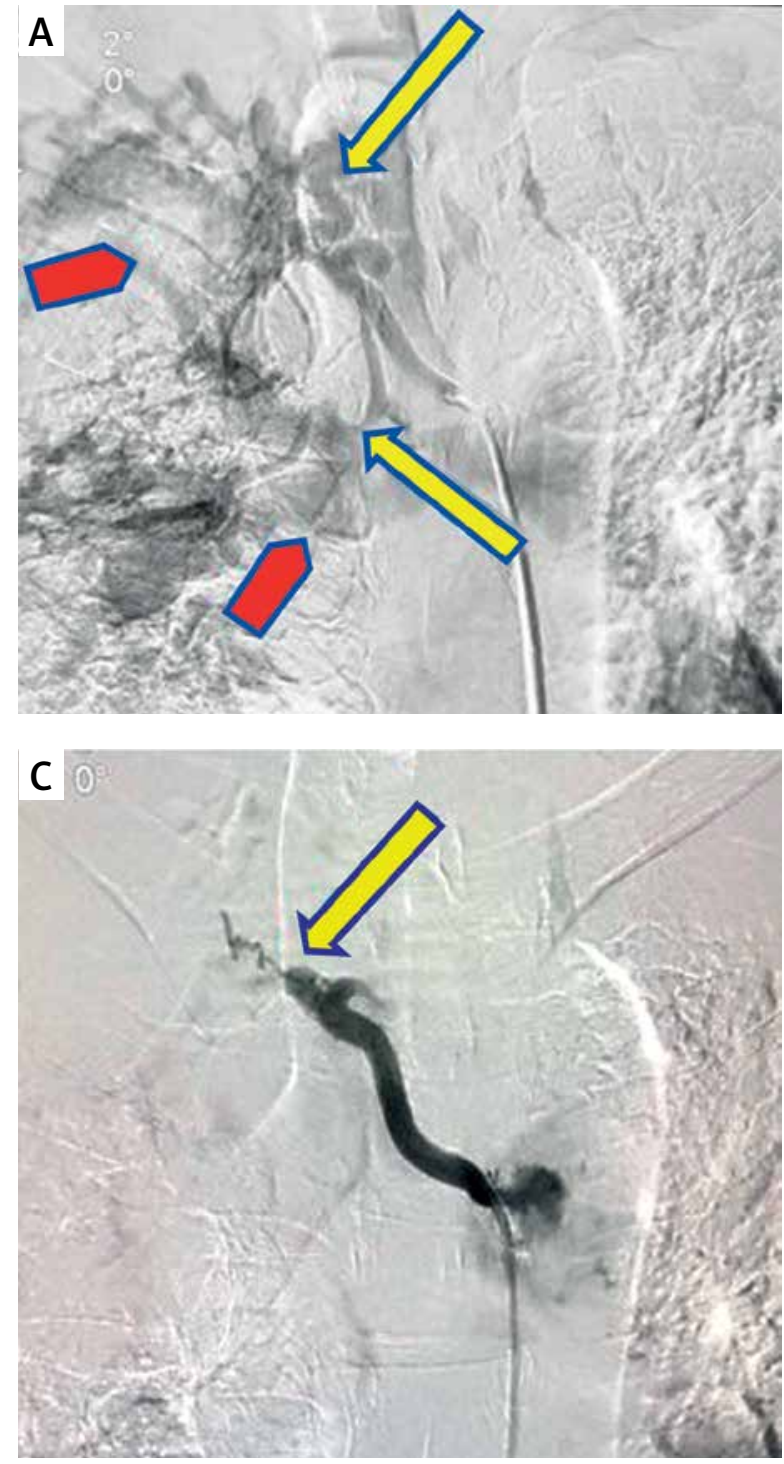

ed on flush aortogram, although a thorough search made on the basis of CTA did reveal collateral circulation that required embolization (Photo 4). A few other authors have also discussed the importance of comprehensive search and complete embolization of the bronchial as well as non-bronchial circulation during the first session itself [3, 9, 13-22].

\section{Choice of embolizing agent and end point determination}

Various embolizing agents have been described in the literature, such as gel foam, PVA particles, $n$-butyl cyanoacrylates and coils, each of them having different merits and demerits. Gel foam is temporary occluder, is less expensive, readily available

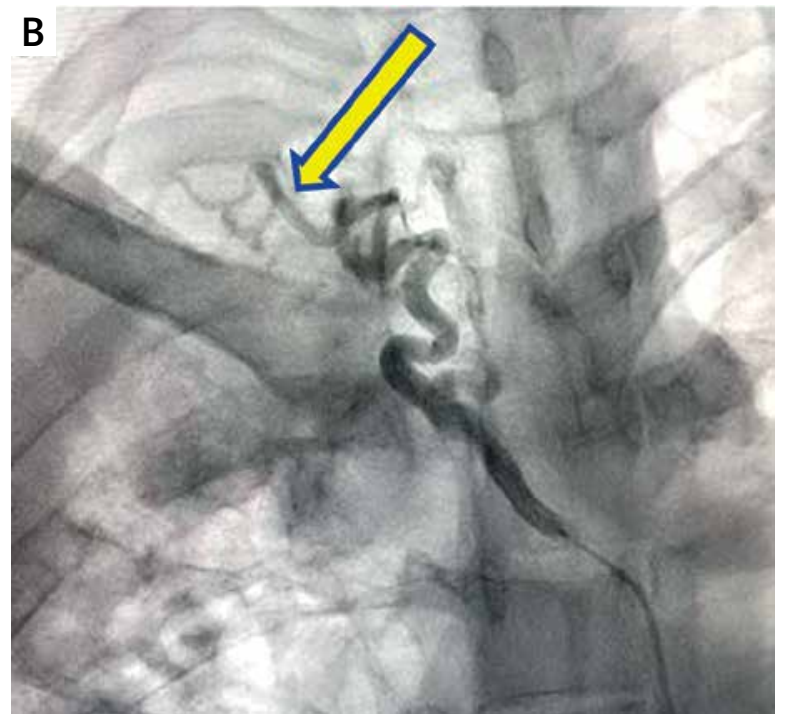

Photo 3. Digital subtraction image (A) shows right intercostobronchial trunk (arrows in A) with large AV shunting seen as early opacification of pulmonary artery (arrowhead in A). Selective catheterization of right intercostobronchial trunk and embolization with PVA particles (decremental size, 500-700 $\mu \mathrm{m}$ followed by 300-500 $\mu \mathrm{m}$ size particles) arrow in C. Repeat angiogram revealed complete non-opacification of abnormal parenchymal blush and non-visualization of shunting (arrow in C)

and requires less operator expertise compared to other agents. $\mathrm{N}$-butyl cyanoacrylates are unique in that they do not require thrombogenic properties of blood and can be offered to patients with coagulopathy disorders. They are among permanent distal occluders with increased rates of necrosis and other complications. Use of coils is limited by high costs to patients, requirement of greater expertise in their deployment along with inability to embolize the parenchymal bed of the abnormality. There is limited possibility of repeat intervention in affected patients after use of coils [8]. PVA particles are considered by far the most suitable embolizing agents as they are readily available and relatively inexpensive. The outcomes are comparable to the use of glue as an 
embolizing agent [9, 22]. In general, the selection of embolizing agent is governed by operator preference and local availability.

In patients having large AV shunts on angiography, we used decremental size of PVA particles. Initial bed embolization was done with $500-700 \mu \mathrm{m}$ particle size followed by embolization of the artery with 300-500 $\mu \mathrm{m}$ size particles. This was done to prevent the possibility of even subclinical pulmonary embolism while achieving arterial occlusion. Smaller size particles were used later because we believed

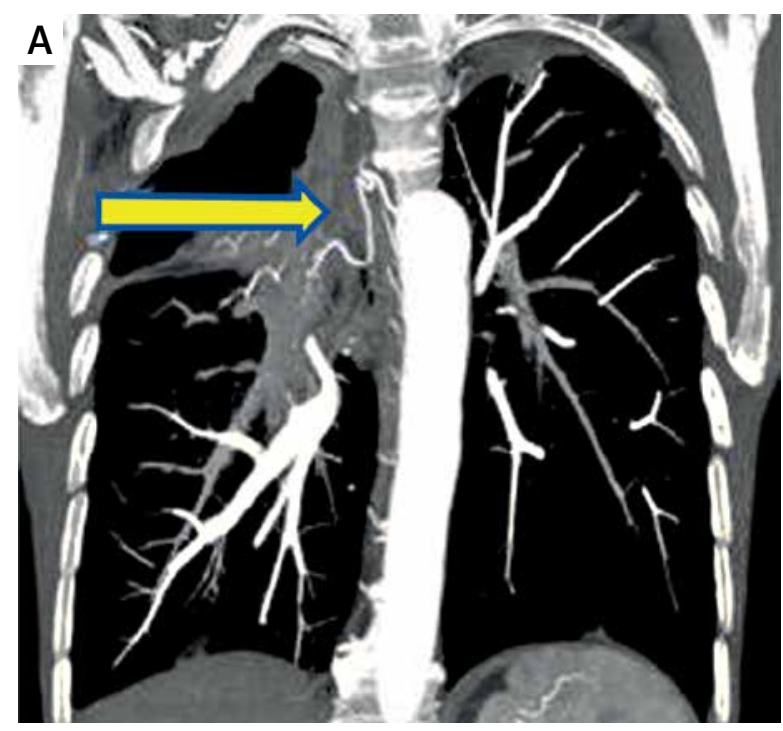

c

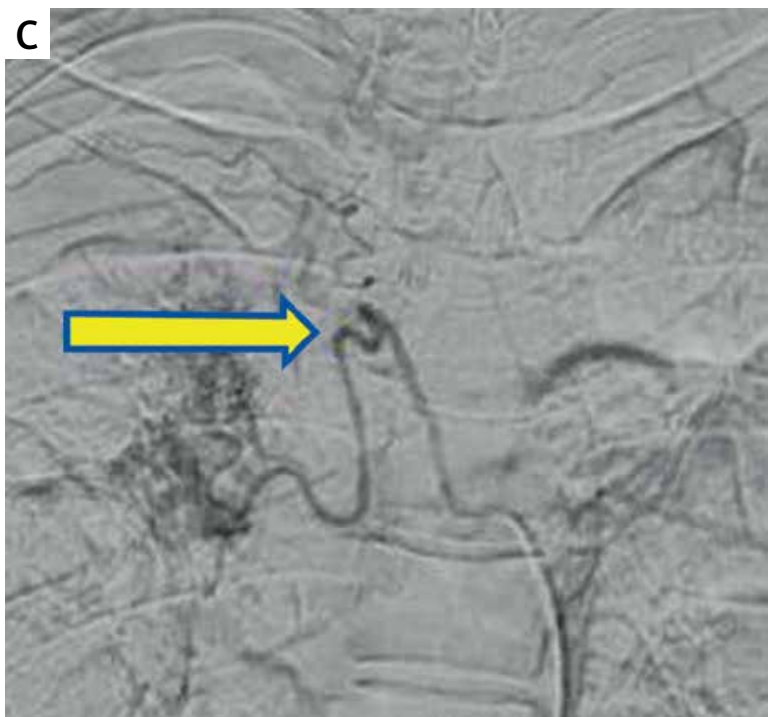

that reflux and resultant non-target embolization of small particles is less harmful as compared to large particles. Also use of smaller size particles eventually never occluded the bed of these large AV shunts, which is safely and expeditiously achieved with larger particles (Photo 3). To the best of our knowledge, use of decremental size of PVA particles, particularly in the setting of large AV shunts, has not been reported in the literature previously.

The use of glue (Histoacryl, Braun Surgical, SA, Rubi, Spain) was limited to the embolization of ar-
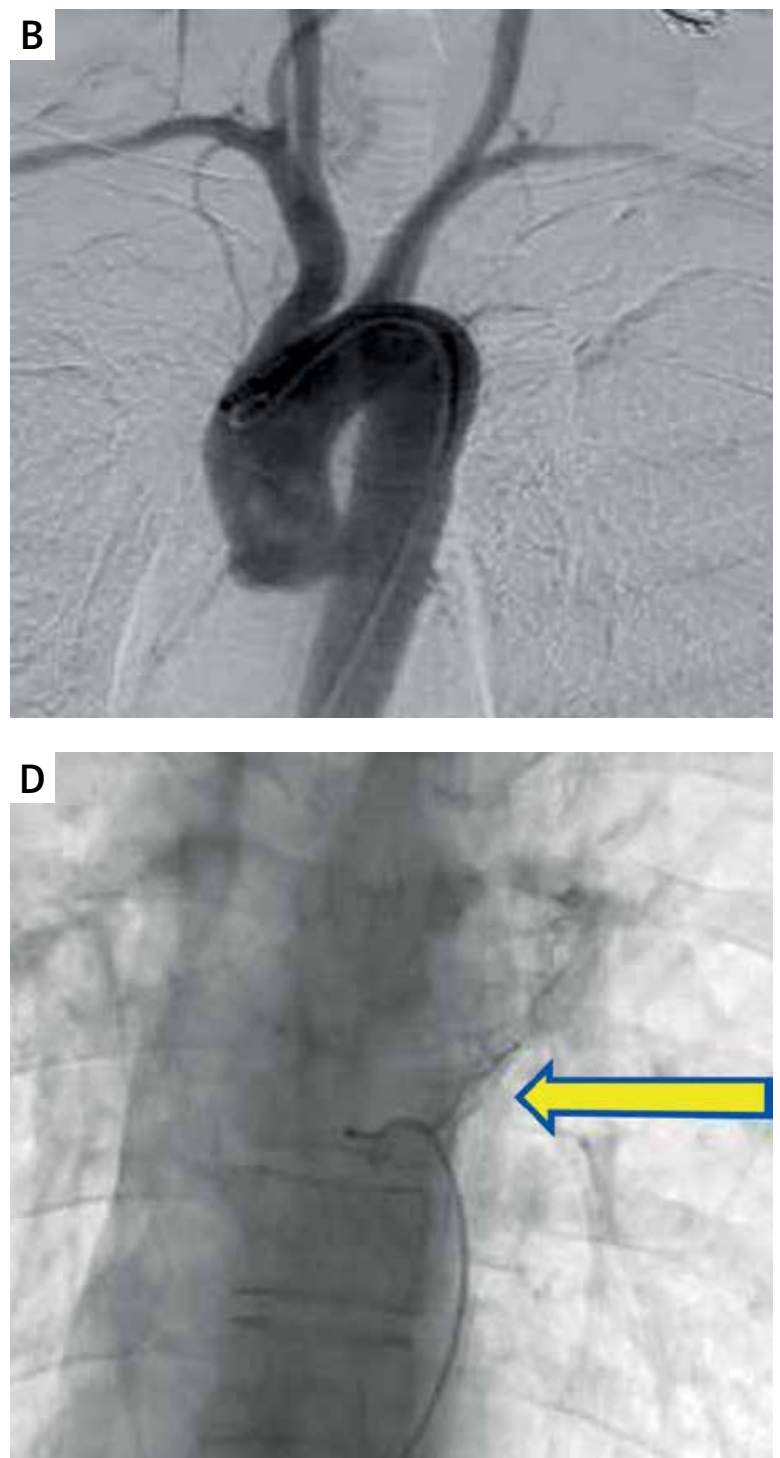

Photo 4. A - Pre-procedure CT angiography depicting presence of a right intercosto-bronchial trunk and associated parenchymal changes. B - Flush aortogram performed during the procedure revealed responsible circulation, but thorough search made on the basis of CT angiography showed presence of right intercosto-bronchial trunk responsible for hemoptysis (C). D - In the same patient, further search revealed small area of parenchymal blush in left lung parenchyma as well arising from left bronchial artery 
teries in which active extravasation was seen at the time of digital subtraction angiography (DSA).

In the present study we achieved technical success in all the patients, while early clinical success was reached in 45 (90\%) patients. On retrospective analysis, it was found that all the 10 patients with recurrence had cavitary lung disease, of which 3 had moderate hemoptysis and 7 had severe hemoptysis at the time of presentation. Our experience was in agreement with various other researchers who in their respective studies also achieved technical success in the range of 95-99\% [7, 11]. We attribute our increased technical success to preprocedure evaluation with CTA as the regular protocol. The clinical success of $80 \%$ using PVA particles along with gel foam was less than that reported in the literature with the use of glue (90-95\%) but reduced incidence of major complications (2\%) far outweighs the amount of clinical success achieved with use of the latter $[14,20]$. The early recurrence rate of $10 \%$ in the present study can be attributed to the inability to embolize third or higher order branches due to extensive collateralization. Late recurrences are postulated to occur due to reconstitution of previously embolized arteries or underlying disease progression causing recruitment of new arteries [10, 15, 21-25]. Of these, 3 patients had large AV shunts and were embolized with decremental PVA particle size as discussed above. The remaining 2 patients with AV shunt did not have any hemoptysis until the 12-month follow-up. A comparison between grade of hemoptysis, embolizing agent used, immediate clinical success and overall recurrence is made in Table I [9, 13, 15, 21, 26, 27].

\section{Complications}

Transient chest/back pain ( $34.5 \%)$ and dysphagia $(-31 \%)$ are amongst the most common complications observed after embolization followed by post-embolization syndrome ( $30 \%)$ comprising fever, leucocytosis and pain [3, 9, 10, 14]. All these complications are frequently self-limiting and usually subside within a week. Contrast media hypersensitivity and puncture site hematomas are now rarely reported with the advent of newer contrast agents and hardware. The most dreaded complications reported are attributed to non-target embolization leading to ischemia of the spinal cord (4.4\%) $[10,13,14,18,22,28]$. Stroke, cortical blindness, bowel ischemia and intermittent claudication together constitute infrequently (2\%) reported complications. Since the development of microcatheters and microwires, the technique of superselective catheterization and use of PVA particles of size 500 $\mu \mathrm{m}$ made complications related to non-target embolization relatively rare. Despite advances, proper counseling and informed consent must be obtained prior to embolotherapy. Two studies have reported spinal cord ischemia induced paraparesis inadvertently as spinal arteries were not visualized even on

Table I. Comparison of clinical parameters, procedural success and recurrence in various studies

\begin{tabular}{|c|c|c|c|c|c|}
\hline References & $\begin{array}{l}\text { Number } \\
\text { of patients }\end{array}$ & Grade of hemoptysis (\%) & $\begin{array}{l}\text { Embolizing } \\
\text { agents }\end{array}$ & $\begin{array}{l}\text { Immediate } \\
\text { success (\%) }\end{array}$ & $\begin{array}{l}\text { Overall } \\
\text { recurrence }\end{array}$ \\
\hline Woo et al., 2013 [9] & 406 & $\begin{array}{l}\text { Massive }-100 \\
\text { Mild to moderate }-0\end{array}$ & $P, C$ & 94 & - \\
\hline Agmy et al., 2013 [13] & 341 & $\begin{array}{c}\text { Massive }-17.5 \\
\text { Mild to moderate }-82.5\end{array}$ & $P, C^{\prime}$ & 95 & 17.6 \\
\hline Bhalla et al., 2015 [15] & 334 & $\begin{array}{c}\text { Massive }-21 \\
\text { Mild to moderate }-79\end{array}$ & P, Ge, Gl & 92 & 14 \\
\hline Lee et al., 2008 [21] & 70 & $\begin{array}{c}\text { Massive }-59 \\
\text { Mild to moderate }-41\end{array}$ & $P$ & 99 & 36 \\
\hline Zhang et al., 1994 [26] & 35 & $\begin{array}{l}\text { Massive }-100 \\
\text { Mild to moderate - } 0\end{array}$ & $\mathrm{Ge}, \mathrm{C}$ & 91.4 & 20 \\
\hline Poyanli et al., 2014 [27] & 140 & $\begin{array}{l}\text { Massive }-69 \\
\text { Mild to moderate }-31\end{array}$ & NA & 98.5 & 10 \\
\hline Present study & 50 & $\begin{array}{l}\text { Moderate to massive }-40 \\
\text { Mild }-10\end{array}$ & P, Ge, Gl & 100 & 20 \\
\hline
\end{tabular}

$P$-PVA, C - Coils, Ge - Gelatin sponge, Gl - Glue, NA - data not available. 
retrospective review of digital subtraction images $[20,26]$. In the present study, in the single case of spinal cord ischemia after the procedure, we could not visualize spinal arteries on DSA images even on retrospective analysis. Further, it was noted to happen in one of the 2 patients who presented with life-threatening hempotysis and CTA could not be performed before the emergency procedure. The patient was referred to PMR (Physical Medicine and Rehabilitation) department to enhance and restore functional ability.

\section{Conclusions}

Bronchial artery embolization is a minimally invasive procedure recognized for primary management of hemoptysis of all grades, and it may also be seen as a bridge to more definitive elective surgical interventions. Preprocedure evaluation with CT angiography can add incremental value by providing a vascular roadmap prior to embolization and predicting technical/clinical outcome of the procedure. Given that there are high chances of recurrence of hemoptysis in affected patients and the need for re-embolization may arise, embolotherapy with PVA particles and gel foam should be preferred over the use of glue and other techniques such as permanent occluders. Decremental particle size usage may be helpful in cases with large AV shunts presenting with hemoptysis.

\section{Conflict of interest}

The authors declare no conflict of interest.

\section{References}

1. Remy J, Voisin C, Dupuis C. Traitement des hemoptysies par embolization de la circulation systemique. Ann Radiol 1974; 17: 5-16.

2. Panda A, Bhalla AS, Goyal A. Bronchial artery embolization in hemoptysis: a systematic review. Diagn Interv Radiol 2017; 23 : 307-17.

3. Anuradha C, Shyamkumar NK, Vinu M, et al. Outcomes of bronchial artery embolization for life-threatening hemoptysis due to tuberculosis and post-tuberculosis sequelae. Diagn Interv Radiol 2012; 18: 96-101.

4. Burke CT, Mauro MA. Bronchial artery embolization. Semin Intervent Radiol 2004; 21: 43-8.

5. Ketai LH, Kirsch J, Kanne JP, et al. ACR Appropriateness Criteria Hemoptysis. J Thorac Imaging 2014; 29: W19-22.

6. Cauldwell EW, Siekert RG, Lininger RE, et al. The bronchial arteries; an anatomic study of 150 human cadavers. Surg Gynecol Obstet 1948; 86: 395-412.
7. Dabo H, Gomes R, Marinho A, et al. Bronchial artery embolisation in management of hemoptysis - a retrospective analysis in a tertiary university hospital. Rev Port Pneumol 2006; 22: 34-8.

8. Sopko DR, Smith TP. Bronchial artery embolization for haemoptysis. Semin Interv Radiol 2011; 28: 48-62.

9. Woo S, Yoon CJ, Chung JW, et al. Bronchial artery embolization to control hemoptysis: comparison of N-butyl-2-cyanoacrylate and polyvinyl alcohol particles. Radiology 2013; 269: 594-602.

10. Chan VL, So LKY, Lam JYM, et al. Major haemoptysis in Hong Kong: aetiologies, angiographic findings and outcomes of bronchial artery embolisation. Int J Tuberc Lung Dis 2009; 13: 1167-73.

11. Yoo DH, Yoon CJ, Kang SG, et al. Bronchial and nonbronchial systemic artery embolization in patients with major hemoptysis: safety and efficacy of N-butyl cyanoacrylate. AJR Am J Roentgenol 2011; 196: W199-204.

12. Swanson KL, Johnson CM, Prakash UBS, et al. Bronchial artery embolization: experience with 54 patients. Chest 2002; 121: 789-95.

13. Agmy GM, Wafy SM, Mohamed SAA, et al. Bronchial and nonbronchial systemic artery embolization in management of hemoptysis: experience with 348 patients. Int Sch Res Not 2013; 26: e263259.

14. Fruchter O, Schneer S, Rusanov V, et al. Bronchial artery embolization for massive hemoptysis: long-term follow-up. Asian Cardiovasc Thorac Ann 2015; 23: 55-60.

15. Bhalla A, Kandasamy D, Veedu P, et al. A retrospective analysis of 334 cases of hemoptysis treated by bronchial artery embolization. Oman Med J 2015; 30: 119-28.

16. Shao H, Wu J, Wu Q, et al. Bronchial artery embolization for hemoptysis: a retrospective observational study of 344 patients. Chin Med J 2015; 12: 58-62.

17. Tom LM, Palevsky HI, Holsclaw DS, et al. Recurrent bleeding, survival, and longitudinal pulmonary function following bronchial artery embolization for hemoptysis in a U.S. adult population. J Vasc Interv Radiol 2015; 26: 1806-13.

18. Rémy J, Arnaud A, Fardou H, et al. Treatment of hemoptysis by embolization of bronchial arteries. Radiology 1977; 122: 33-7.

19. Yu-Tang Goh P, Lin M, Teo N, En Shen Wong D. Embolization for hemoptysis: a six-year review. Cardiovasc Intervent Radiol 2002; 25: 17-25.

20. van den Heuvel MM, Els Z, Koegelenberg CF, et al. Risk factors for recurrence of haemoptysis following bronchial artery embolisation for life-threatening haemoptysis. Int J Tuberc Lung Dis 2007; 11: 909-14.

21. Lee S, Chan JWM, Chan SCH, et al. Bronchial artery embolisation can be equally safe and effective in the management of chronic recurrent haemoptysis. Hong Kong Med J 2008; 14: 14-20.

22. Chan VL, So LKY, Lam JYM, et al. Major haemoptysis in Hong Kong: aetiologies, angiographic findings and outcomes of bronchial artery embolisation. Int J Tuberc Lung Dis 2009; 13: 1167-73.

23. Shin BS, Jeon GS, Lee SA, Park MH. Bronchial artery embolisation for the management of haemoptysis in patients with pulmonary tuberculosis. Int J Tuberc Lung Dis 2011; 15: 1093-8.

24. Lorenz J, Sheth D, Patel J. Bronchial artery embolization. Semin Intervent Radiol 2012; 29: 155-60. 
25. Rabkin JE, Astafjev VI, Gothman LN, Grigorjev YG. Transcatheter embolization in the management of pulmonary hemorrhage. Radiology 1987; 163: 361-5.

26. Zhang JS, Cui ZP, Wang MQ, Yang L. Bronchial arteriography and transcatheter embolization in the management of hemoptysis. Cardiovasc Intervent Radiol 1994; 17: 276-9.

27. Poyanli A, Acunas B, Rozanes I, et al. Endovascular therapy in the management of moderate and massive haemoptysis. $\mathrm{Br}$ J Radiol 2007; 80: 331-6.

28. Ramakantan R, Bandekar VG, Gandhi MS, et al. Massive hemoptysis due to pulmonary tuberculosis: control with bronchial artery embolization. Radiology 1996; 200: 691-4.

Received: 13.09.2019, accepted: 25.10.2019. 